\title{
ALK germline mutations in patients with neuroblastoma: a rare and weakly penetrant syndrome
}

\author{
Franck Bourdeaut ${ }^{1,2,3}$, Sandrine Ferrand ${ }^{4}$, Laurence Brugières ${ }^{5}$, Marjorie Hilbert ${ }^{6}$, Agnès Ribeiro $^{4}$, \\ Ludovic Lacroix ${ }^{7}$, Jean Bénard ${ }^{7}$, Valérie Combaret ${ }^{8}$, Jean Michon ${ }^{1}$, Dominique Valteau-Couanet ${ }^{5}$, \\ Bertrand Isidor $^{9}$, Xavier Rialland ${ }^{10}$, Maryline Poirée ${ }^{11}$, Anne-Sophie Defachelles ${ }^{12}$, Michel Peuchmaur ${ }^{13}$, \\ Gudrun Schleiermacher ${ }^{1,2}$, Gaëlle Pierron ${ }^{4}$, Marion Gauthier-Villars ${ }^{14}$, Isabelle Janoueix-Lerosey ${ }^{2}$ and \\ Olivier Delattre ${ }^{\star, 2,4}$ on behalf of the Comité Neuroblastome of the Société Francaise de Cancérologie \\ de l'Enfant
}

\begin{abstract}
Neuroblastic tumours may occur in a predisposition context. Two main genes are involved: $P H O X 2 B$, observed in familial cases and frequently associated with other neurocristopathies (Ondine's and Hirschsprung's disease); and ALK, mostly in familial tumours. We have assessed the frequency of mutations of these two genes in patients with a presumable higher risk of predisposition. We sequenced both genes in 26 perinatal cases (prebirth and $<1$ month of age, among which 10 were multifocal), 16 multifocal postnatal ( $>1$ month) cases, 3 pairs of affected relatives and 8 patients with multiple malignancies. The whole coding sequences of the two genes were analysed in tumour and/or constitutional DNAs. We found three $A L K$ germline mutations, all in a context of multifocal tumours. Two mutations (T1151R and R1192P) were inherited and shared by several unaffected patients, thus illustrating an incomplete penetrance. Younger age at tumour onset did not seem to offer a relevant selection criterion for $A L K$ analyses. Conversely, multifocal tumours might be the most to benefit from the genetic screening. Finally, no PHOX2B germline mutation was found in this series. In conclusion, ALK deleterious mutations are rare events in patients with a high probability of predisposition. Other predisposing genes remain to be discovered.
\end{abstract} European Journal of Human Genetics (2012) 20, 291-297; doi:10.1038/ejhg.2011.195; published online 9 November 2011

Keywords: ALK; neuroblastoma; predisposition

\section{INTRODUCTION}

Most cancers occurring in children are thought to be sporadic and a genetic predisposition is rarely evoked. However, some particular clinical features are more suggestive of a genetic susceptibility and justify further investigation. Familial aggregations of cancers are among the most suggestive situations, having lead, for example, to the identification of the Li-Fraumeni syndrome. ${ }^{1}$ However, random familial aggregations of sporadic cancers are not so unusual. Thus, the suspicion of familial susceptibility is more justified when several individuals develop the same malignancy. The paradigmatic example in paediatric cancers is the familial form of retinoblastoma due to hereditary mutations in $R B 1,{ }^{2}$ in which multifocal disease is a common observation. Similarly, bilateral Wilms tumours are indicative of a predisposition, occasionally related to $11 \mathrm{p} 15$ abnormality with or without Beckwith-Wiedemann phenotype, ${ }^{3,4}$ which is the paradigmatic imprinting defect associated with neoplasia. ${ }^{5}$ Therefore, a multifocal presentation is sufficient per se to suggest a predisposition syndrome, especially when it is observed with any kind of developmental disease. ${ }^{4}$ Finally, a young age at diagnosis is also indicative of a higher probability of carrying a germline mutation. Such a positive correlation between the presence of a germline mutation and the youngest age at diagnosis has been proven for several predisposing genes, such as RB1 in retinoblastoma, SMARCB1 in rhabdoid tumours. Another example come from the mutations in SUFU (a tumour suppressor gene controlling the Sonic hedgehog signalling) which most remarkably predispose to early onset desmoplastic medulloblastomas, before 3 years of age. ${ }^{6,7}$

Peripheral neuroblastic tumours are tumours of neural crest origin that mostly affect children before 5 years of age. The young age at diagnosis lead Knudson and Strong ${ }^{8}$ to propose that neuroblastic tumours might frequently arise in a predisposition context. Accordingly, familial cases, although rare, confirmed the hypothesis of germline mutations being responsible for at least a subset of those tumours. ${ }^{9,10}$ Recently, mutations in two genes have been identified as predisposing causes for neuroblastic tumours. First, mutations in $P H O X 2 B$, a gene expressed in the developing peripheral autonomous nervous system, were observed in children with neuroblastic tumours in a syndromic context of Ondine's curse and/or Hirschsprung

${ }^{1}$ Institut Curie, Département de pédiatrie, Paris, France; ${ }^{2}$ INSERMU830, Laboratoire de Génétique et Biologie des cancers, Institut Curie, Paris, France; ${ }^{3} \mathrm{CHU}$ Nantes, Service d'Hémato-Oncologie Pédiatrique, Nantes, France; ${ }^{4}$ Institut Curie, Unité de génétique somatique, Paris, France; ${ }^{5}$ Institut Gustave Roussy, Département de pédiatrie, Villejuif, France; ${ }^{6}$ Service d'Hémato-Oncologie Pédiatrique, Université de Bordeaux \& CHU de Bordeaux, Bordeaux, France; ${ }^{7}$ Institut Gustave Roussy, Département de Biologie et Pathologie médicales, Villejuif, France; ${ }^{8}$ Centre Léon Bérard, Laboratoire de recherche translationnelle, Lyon, France; ${ }^{9} \mathrm{CHU}$ Nantes, Service de Génétique Médicale, Nantes, France; ${ }^{10} \mathrm{CHU}$ Angers, Service d'Hémato-Oncologie Pédiatrique, Angers, France; ${ }^{11} \mathrm{CHU}$ Nice, Service d'Hémato-Oncologie Pédiatrique, Nice, France; ${ }^{12} \mathrm{Centre}$ Oscar Lambret, Département de pédiatrie, Lille, France; ${ }^{13}$ Université Paris 6, Hôpital Robert Debré, Service d'anatomie pathologique, Paris, France; 14Institut Curie, Département d'oncogénétique, Paris, France

*Correspondence: Dr O Delattre, INSERMU830, Laboratoire de Génétique et Biologie des Cancers, Institut Curie, 26 rue d’Ulm, 75348 Paris Cedex 05, France.

Fax: +33 1542666 30; Tel: +33 1562466 79; E-mail: olivier.delattre@curie.fr

Received 13 July 2011; revised 15 September 2011; accepted 22 September 2011; published online 9 November 2011 
disease. ${ }^{11-13}$ Subsequent studies showed that $P H O X 2 B$ mutations could also be observed in non-syndromic familial cases, and even as de novo mutations in apparently sporadic tumours. ${ }^{14-17}$ More recently, constitutional mutations in the $A L K$ gene have been shown to be the predominant genetic cause of familial neuroblastomas. ${ }^{18,19}$ Furthermore, somatically acquired $A L K$ mutations are evidenced in $\sim 7 \%$ of sporadic tumours, but, again, some of those apparently sporadic tumours actually arise in a context of constitutional mutation, either inherited or de novo. ${ }^{20-23}$ Hence, the actual incidence of $P H O X 2 B$ and $A L K$ germline mutations may be highly underestimated if one only considers familial recurrences of neuroblastic tumours to initiate a constitutional screening.

In the present work, we have addressed the frequency of $A L K$ germline mutations in children who developed a neuroblastoma, a ganglioneuroblastoma or a ganglioneuroma with clinical features highly suggestive of a predisposition context. Based on previous experiences and literature review, we selected, as good candidates for having a predisposition, children with perinatal diagnosis (before birth and up to 1 month of age), ${ }^{24}$ multifocal disease, ${ }^{25}$ second malignancy, ${ }^{26}$ and familial recurrence of neuroblastic tumours. Our work aims at giving a better insight for genetic counselling and further document the incidence and the penetrance of $A L K$ mutations in patients with neuroblastic tumours.

\section{MATERIALS AND METHODS}

\section{Selection of the study population}

We first retrospectively reviewed the clinical database from the tumour banks of Institut Curie and Institut Gustave Roussy; the study period was 1998-2010. We first selected patients in whom the diagnosis of a neuroblastic tumour was made either before birth by antenatal ultrasonography or within the first month of life (perinatal tumour) (Table 1). We also included patients harbouring a multifocal disease; the multifocal status of the disease consisted in (i) significant enlargement and/or tumour mass of both adrenal glands, (ii) synchronous tumours emerging from at least two distant sites of the autonomous nervous system, apart from the adrenal gland, and (iii) metachronous neuroblastic tumours occurring at different sites (Table 2).

Familial cases were also explored. Three new French familial cases were identified in which siblings (families 1 and 2) or first cousins (family 3) were affected by neuroblastic tumours (Figure 3). In families 1 and 2, interestingly, the thoracic neuroblastic tumours of the two elder boys were fortuitously diagnosed on a chest X-ray performed for a febrile cough.

Finally, we included patients who developed multiple malignancies including one neuroblastic tumour (Table 3).

Table 1 Results of $A L K$ mutation screening in sporadic perinatal and multifocal postnatal cases

\begin{tabular}{|c|c|c|c|c|c|c|c|c|}
\hline & \multicolumn{2}{|c|}{ Localized } & \multicolumn{2}{|c|}{$M S$} & \multicolumn{2}{|c|}{$M$} & \multirow[b]{2}{*}{ Unknown } & \multirow[b]{2}{*}{ Total } \\
\hline & Multi. & Uni. & Multi. & Uni. & Multi. & Uni. & & \\
\hline \multicolumn{9}{|l|}{ Perinatal } \\
\hline Tum. & $1 / 4$ & $1 / 15$ & $0 / 6$ & $0 / 4$ & $0 / 0$ & $1 / 2$ & $0 / 5$ & $2 / 36$ \\
\hline Const. & 1 & 0 & 0 & 0 & 0 & 0 & 0 & 1 \\
\hline \multicolumn{9}{|c|}{$>1$ month } \\
\hline Tum. & $0 / 3$ & & $2 / 3$ & & & & $0 / 0$ & $2 / 6$ \\
\hline Const. & 0 & & 2 & & & & 0 & 2 \\
\hline \multicolumn{9}{|c|}{ Total of const. mutations } \\
\hline & $1 / 7$ & $0 / 15$ & $2 / 9$ & $0 / 4$ & $0 / 0$ & $0 / 2$ & $0 / 5$ & $3 / 42 *$ \\
\hline & \multicolumn{2}{|c|}{$1 / 22$} & \multicolumn{2}{|c|}{$2 / 13$} & \multicolumn{2}{|c|}{$0 / 2$} & $0 / 5$ & \\
\hline
\end{tabular}

\section{Tumour and constitutional DNA analyses}

We first assessed the status of $A L K$ mutations in tumour DNAs. Mutation screening in the tumours were made according to the French Huriet law regarding the biological research on human tissue samples.

A consultation with a geneticist was proposed in case of a familial recurrence, multifocal disease and multiple malignancies; it was also proposed when a mutation in the $A L K$ gene was observed in the tumour DNA. Informed consent was obtained from the parents or legal tutor of the affected children.

Tumour cell content was assessed by cryosection on frozen samples, to help in the interpretation of a minor allele in case of abundant stroma.

Direct sequencings of the 29 exons of $A L K$ and the 3 exons of $P H O X 2 B$ were performed using the classical Sanger procedures. Primers sequences and PCR conditions have been published elsewhere and are available on request. ${ }^{18,27}$ Mutations were a priori considered deleterious when leading to obvious

Table 2 Summary of clinical data for multifocal cases

\begin{tabular}{|c|c|c|c|c|c|c|}
\hline $\begin{array}{l}\text { Patient } \\
\text { no. }\end{array}$ & Stage & Location & Histology & Occurrence & PHOX2B & $A L K$ \\
\hline \multicolumn{7}{|c|}{ Neonatal case } \\
\hline mNB1 & L & Adre $\times 2+$ thor & $\mathrm{NB}+\mathrm{GNB}$ & Syn & wt & wt \\
\hline mNB2 & L & $\begin{array}{l}\text { Adre } \times 2+ \\
\text { cerv+thor }\end{array}$ & $\mathrm{NB}+\mathrm{NB}+\mathrm{NB}$ & Meta & wt & $R 1275 Q^{*}$ \\
\hline $\mathrm{mNB3}$ & L & Adre $x 2$ & $N B+N B$ & Meta & wt & wt \\
\hline $\mathrm{mNB} 4$ & L & Adre $\times 2+$ sub/c & $\mathrm{NB}+\mathrm{GN}$ & Meta & wt & wt \\
\hline mNB5 & MS & Adre $x 2$ & NB, nos & Syn & wt & wt \\
\hline $\mathrm{mNB6}$ & MS & Adre $x 2$ & NB, nos & Syn & wt & wt \\
\hline $\mathrm{mNB7}$ & MS & Adre $x 2$ & NB, nos & Syn & wt & wt \\
\hline $\mathrm{mNB8}$ & MS & Adre $x 2$ & NB, nos & Syn & wt & wt \\
\hline mNB9 & MS & Adre $\times 2$ & NB, nos & Syn & wt & wt \\
\hline $\mathrm{mNB} 10$ & MS & Adre $\times 2$ & NB, nos & Syn & wt & wt \\
\hline \multicolumn{7}{|c|}{ Postnatal cases } \\
\hline mNB11 & $\mathrm{L}$ & Adre+thor & $\mathrm{NB}+\mathrm{NB}$ & Syn & wt & wt \\
\hline $\mathrm{mNB12}$ & $\mathrm{L}$ & Adre+cerv & $\mathrm{NB}+\mathrm{GNB}$ & Syn & wt & wt \\
\hline $\mathrm{mNB} 13$ & $\mathrm{~L}$ & Adre $x 2$ & $\mathrm{NB}+\mathrm{GN}$ & Syn & wt & wt \\
\hline mNB14 & MS & Adre+sub/c & $\mathrm{NB}+\mathrm{GN}$ & Meta & wt & R1192P* \\
\hline mNB15 & MS & Pelv+sub/c & $N B+G N$ & Meta & 676insG & wt \\
\hline $\mathrm{mNB} 16$ & MS & Adrex2+retrop & $\mathrm{NB}+\mathrm{NB}$ & Syn & wt & T1151R* \\
\hline
\end{tabular}

Abbreviations: mNB, multifocal neuroblastoma; MS, Pepper syndrome; L, localized; Adre, adrenal gland; thor, thoracic; cerv, cervical; pelv, Pelvic; retrop, retroperitoneal; sub/c, subcutaneous; NB, neuroblastoma; GNB, ganglioneuroblastoma; GN, ganglioneuroma; nos, Not otherwise specified (no biopsy); Syn, synchronous; Meta, metachronous; wt, wild type.

*Mutation found in the germline.

Table 3 Clinical and genetic features of patients with multiple neoplasia including a neuroblastic tumour

\begin{tabular}{lll}
\hline First tumour & Second tumour & Genetic results \\
\hline $\begin{array}{l}\text { Unilateral retinoblastoma, } \\
16 \text { months }\end{array}$ & $\begin{array}{l}\text { Adrenal ganglioneuroblas- } \\
\text { toma, 9 years }\end{array}$ & ALK, RB1 wt \\
Unilateral nephroblastoma, & Adrenal neuroblastoma, 9 & No 11p15 LOI, ALK, \\
9 months & months & CDKN1C wt \\
Neuroblastoma, 3 years & Rhabdoid tumour, 22 years & $A L K$ wt, SMARCB1 wt \\
Abdominal NB, 5years & Desmoid tumour, 27 years & $A L K$ wt \\
Melanoma, 22 years & Adrenal neuroblastoma, & $A L K, C D K 4$, \\
& 30years & $C D K N 2 A$ wt \\
Neuroblastoma, 9 months & Nerve sheath tumour, & $A L K$ wt \\
& 28years & \\
Neuroblastoma, 8 years & Liposarcoma, 28years & $A L K$ wt \\
Abdominal neuroblastoma, & Medulloblastoma, 3years & $A L K$ wt \\
3years & &
\end{tabular}

Abbreviations: RB, retinoblastoma; wt, wild type; LOI, loss of imprinting. 
impairment of the protein (premature stop codon and frameshift) or when affecting the functional domains of the protein (tyrosine kinase of $A L K$, and homeodomain or polyalanine stretch of $P H O X 2 B$ ) on highly conserved residues (unique amino-acid substitution).

\section{Familial analyses}

Germline analyses of the relatives were investigated upon the parents' request and with the appropriate information to the siblings. Informed consents of the individuals or their legal tutors were obtained for all germline analyses. In case of a germline mutation in asymptomatic relatives of the index case, a chest $\mathrm{X}$-ray and an abdominal ultrasonography were performed to evidence a silent benign neuroblastic tumour.

\section{Array CGH on tumour DNA}

Three tumours were analysed on a homemade BAC arrays. Subsequent analyses were performed on NimbleGen $4 \times 72 \mathrm{~K}$ arrays. Genomic DNA extracted with Phase Lock Gel Light (Eppendorf, Le Pecq, France) and was analysed versus normal reference DNA. In all, $700 \mathrm{ng}$ of each sample was labelled and cohybridized to the NimbleGen $4 \times 72 \mathrm{k}$ arrays according to the manufacturer's protocol (Roche NimbelGen, Madison, WI, USA). Arrays were washed and then scanned on a GenePix 4000B scanner using GenePix 6.0 Software (Axon, Sunnyvale, CA, USA). Raw data were normalized using NimbleScan v2.5 Software (Roche NimbleGen) and then processed using NimbleScan Software using the segMNT algorithm to obtain data with a resolution of $100 \mathrm{pb}$. The data were then visualized with SignalMap v1.9 (Roche NimbelGen) before analysis. Normalized data are available on http://microarrays.curie.fr/.

\section{RESULTS}

Perinatal ( $<1$ month) cases

Thirty-six perinatal cases were analysed. Among those, ten newborns showed a multifocal disease, involving both adrenal glands in seven cases. None of the seven bilateral adrenal neuroblastomas showed a mutation. Conversely, a c.3824G > A (p.Arg1275Gln, R1275Q) mutation was found both in the tumours and in the germline of a child affected by multiple adrenal and extra-adrenal neuroblastomas (mNB2) (Figure 1b). This initially trifocal tumour (cervical, mediastinal, and adrenal) was revealed by dyspnoea at 2 weeks of age. After successful initial treatment, the child developed a controlateral adrenal tumour and another fifth abdominal retroperitoneal tumour at 9 months of age. Array CGH performed on three different tumours showed clearly distinct profiles (Figure 1a). Interestingly in this patient, the Meckel diverticulitis has been ressected for acute painful inflammation; an unexpected hyperplasia of myenteric plexus was noticed (Figure 1c), a phenomenon that could eventually be somewhat related to the sympathetic tumours given the neural crest origin of the myenteric plexus, despite the neurons harboured a normal morphology. The child is doing well without disease with a 10 -year follow-up.

Apart from those 10 multifocal cases, 26 unifocal perinatal cases were also analysed (Table 1). Two other mutations were found in those latter cases: a p.Pro238del in frame deletion and a c.3824G $>$ A (p.Arg1275Gln, R1275Q) mutation. None of these two variants were confirmed in the germline.

No PHOX2B mutation was found in any of those perinatal cases.

\section{Multifocal postnatal ( $>1$ month) cases}

The main features of all multifocal cases are reported in Table 2. Among the six postnatal patients with multifocal tumours, ALK mutations were found in two tumours. For one patient, a c.3452C > G (p.Thr1151Arg, T1151R) was also present in the germline (mNB16). The girl developed a stage MS neuroblastoma; one adrenal gland was obviously enlarged, the second showed multiple calcifications compatible with tumour involvement and another bulky mass was seen in the paravertebral thoracic region. After initial treatment, the tumour relapsed with bone metastasis, leading to intensification of the treatment and autologous haematopoietic stem cells infusion. She is alive without disease with a 5-year follow-up.

The second patient had an adrenal stage $M$ neuroblastoma diagnosed at 6 months, and developed multiple paravertebral and subcutaneous ganglioneuromas, arising from nervous ganglia and fibers at cervical, thoracic, and pelvic levels, in successive waves up to 6 years of age (mNB14). A c.3575G >C (p.Arg1192Pro, R1192P) $A L K$ mutation was evidenced in all tumours tested and in the lymphocytes, thus confirming the germline predisposing context.

No $A L K$ mutation was found in the other multifocal cases. However, in one patient with a pelvic stage $M$ neuroblastoma, and multiple metachronous subcutaneous, para-uterine and intestinal ganglioneuromas (patient mNB15), the PHOX2B sequencing revealed a 676 ins $G$ mutation. The variation was found in ganglioneuromas and neuroblastoma, but not in the lymphocytes, compatible with a mosaicism. Other tumours did not show any $P H O X 2 B$ mutation.

\section{Familial cases}

Three familial tumours were analysed from three different pedigrees (Figure 2). One ALK c.3522C >A (p.Phe1174Leu, F1174L) was observed (patient F2.2) in the tumour DNA. This variant was not found at the germline level. No mutation in $A L K$ was seen in the other patients. No mutation was found either in the three exons of $P H O X 2 B$.

\section{Multiple cancers}

Eight patients were tested because they have developed multiple cancers, including one neuroblastic tumour (Table 3). No mutation was found in $A L K$ sequence, neither in $P H O X 2 B$.

\section{$A L K$ mutations in the relatives of germline mutation carriers with} neuroblastoma

While the R1275Q was proven to be de novo, the R1192P and T1151R variants were inherited from asymptomatic parents and shared by three and five asymptomatic relatives, respectively (Figure 3). In particular, for the asymptomatic siblings of index cases with a germline mutation, ultrasonography and chest X-rays revealed no tumour so far.

\section{Array CGH on multifocal cases}

To look for potential common chromosomal alterations indicating a putative predisposition locus, array CGH was performed on tumour DNA from distinct sites in three multifocal cases showing no germline $A L K$ nor $P H O X 2 B$ mutation (cases $\mathrm{mNB} 3, \mathrm{mNB} 11$, and $\mathrm{mNB} 12$; Figure 4). The different tumours arising in the same patient showed common gains and deletions but, more interestingly, also specific alterations, suggesting two independent evolutions. The coexistence of several neuroblastic tumours with different genomic background convincingly demonstrated the predisposition context for these patients. However, no remarkable alterations could straightforward orientate to any particular predisposition locus.

\section{DISCUSSION}

To delineate the proportion of those tumour mutations that are also present in the germline is of importance in clinical practice for proper genetic counselling; hence, finding relevant criteria to identify the tumours that arise in a predisposing context would be highly helpful. In the present study, we expected to identify the patients with apparently sporadic tumours who are the most prone to benefit from a germline analysis; with this aim, we specifically studied children showing multifocal neuroblastomas, early occurrence of the 

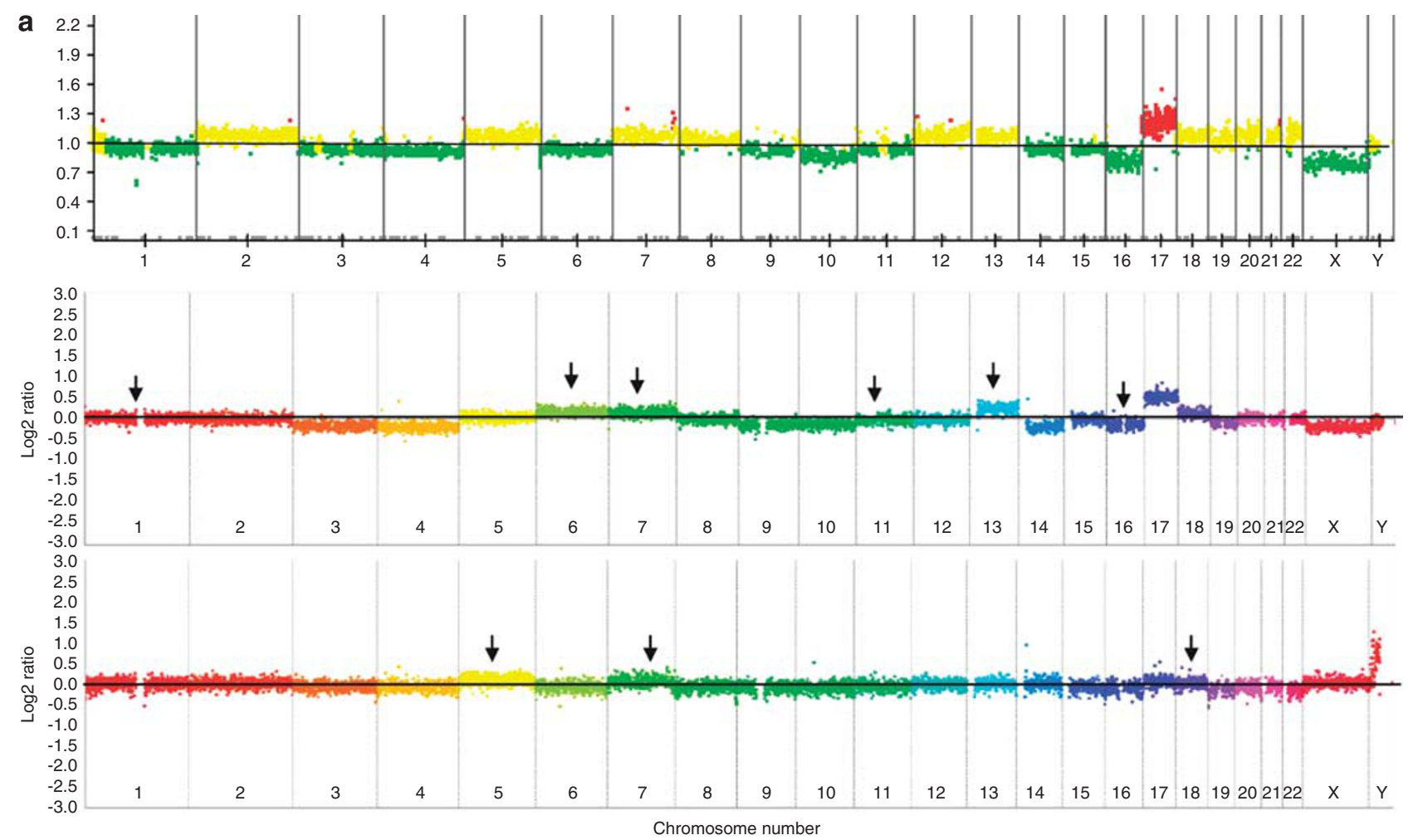

b

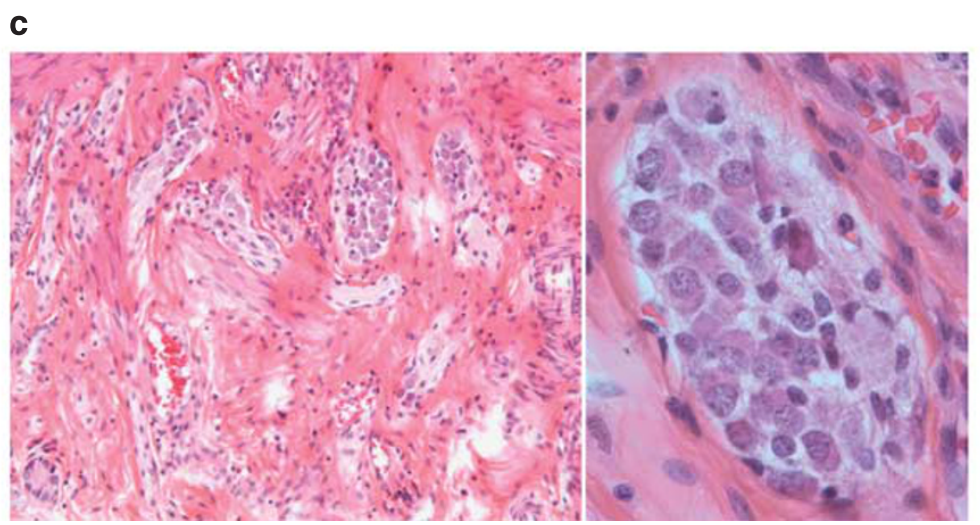

Figure 1 Clinical and genetic features observed in neonatal case (mNB2) with a c.3824G >A (p.Arg1275GIn) mutation. (a) Array CGH on cervical (BAC array), adrenal and retrohepatic tumours (NimbleGen $4 \times 72 \mathrm{~K}$ ) from top to bottom. On BAC arrays, yellow indicates normal copy number, red indicates a chromosomal gain, and green a chromosomal loss. On Nimblegen arrays, colours indicate each a different chromosome. Arrows show the chromosomal copy number statuses that differ between each of the two bottom arrays and the upper one. (b) Nucleotide sequence of exon 25 showing the c.3824G>A (p.Arg1275Gln). (c) Haematoxylin Eosin Safran (HES) on Meckel diverticle showing hyperplasia of the myenteric plexus (left panel), without neoplastic feature (right panel).

tumour or multiple malignancies. Our study concludes that this strategy does not dramatically increase the discovery rate of germline ALK or $P H O X 2 B$ mutations.

Strikingly, previous results from non-familial neuroblastomas have shown a weak incidence of germline mutations in apparently sporadic cases: out of 51 patients showing an $A L K$ mutation in the tumour that was also tested in the germline, 4 only have indeed an $A L K$-related predisposition. ${ }^{18,19,21,23}$ These preliminary results suggested that no more than $8 \%$ of patients with a mutation in the tumour might have a predisposing context. In a recent large meta-analysis, the frequency of somatic $A L K$ mutations was estimated to be $6.9 \%$ out of 709 neuroblastic tumours. Altogether, and although never properly assessed in a systematic screening, this suggests a very weak frequency for $A L K$ germline mutations among all patients with sporadic neuroblastoma. Hence, the low incidence of $A L K$ germline mutation that we have found in sporadic tumours in this study $(3 / 42)$ is still to be interpreted in the light of the ratio extrapolated from published data. However, as previously observed for $P H O X 2 B$, we assume that $A L K$ germline mutation might account for a very limited number of apparently sporadic cases.

In detail, only $1 / 36$ perinatal cases showed an $A L K$ germline mutation. In our cases of $A L K$ germline mutation, neuroblastoma was diagnosed at 2 weeks, 3 months and 6 months of age, compared with $0.6,1.2^{22}$ and $8.2^{19}$ years in the previously reported cases for which the age is available. Altogether, these observations suggest that young age at onset and perinatal tumour development in particular, is not a major relevant criterion to indicate a higher risk for $A L K$-related predisposition. 

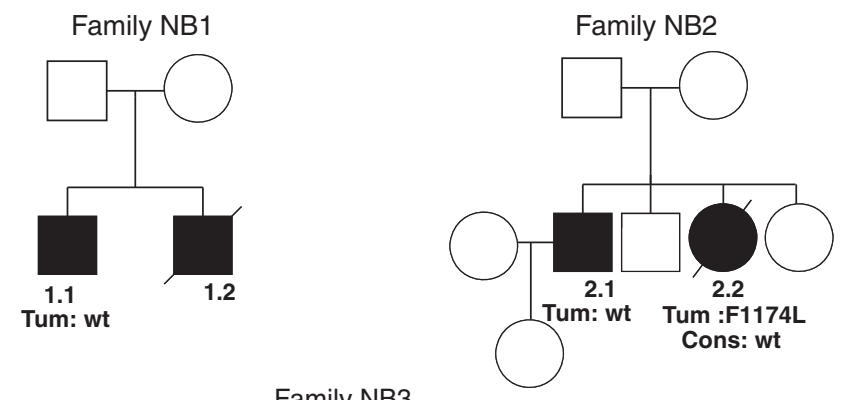

Family NB3

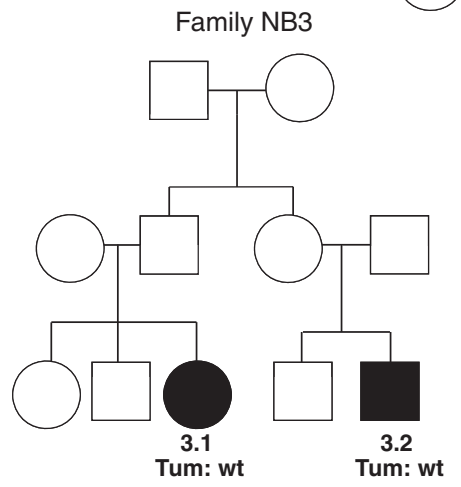

Figure 2 Pedigrees of familial neuroblastic tumours without neither PHOX2B nor ALK mutation. Tum, mutation found in the tumour DNA; Cons, status of $A L K$ gene in the germline; wt, wild type.
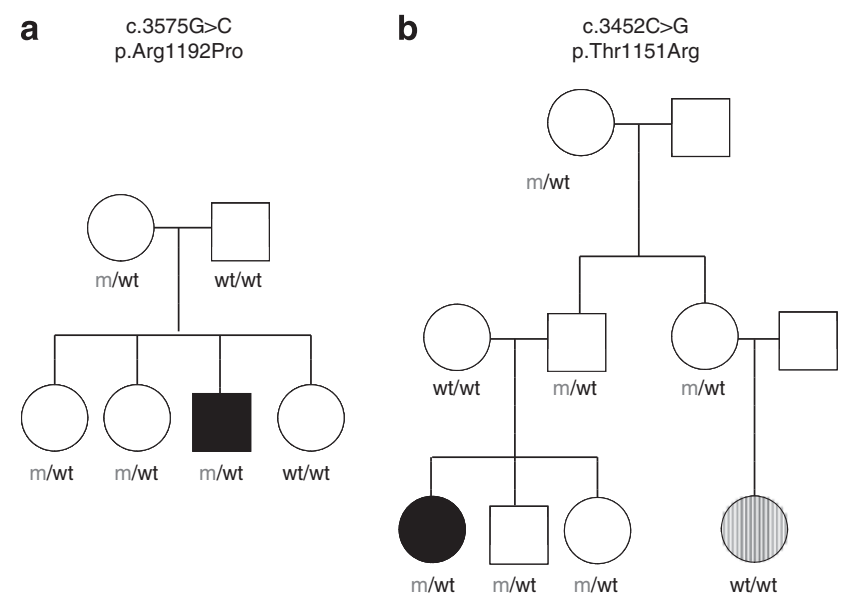

Figure 3 Pedigrees of index cases with inherited germline ALK mutations (mNB14 and mNB16): full dark indicates the children with neuroblastoma; dash indicates retinoblastoma that has been linked in this girl to a de novo constitutional RB1 mutation. m, mutated; wt, wild type.

Moreover, this mutated neonatal case could be clearly distinguished from other perinatal patients because of multiple metachronous neuroblastomas. Considering that 3/16 multifocal apparently sporadic cases in our series show an $A L K$ germline mutation, this particular clinical presentation might be considered as the most strongly suggestive of an $A L K$-related predisposition in absence of familial history. However, from a clinical point of view, making an indubitable difference between metastasis and multiple primary tumours is most often challenging. Although not categorical criterion (i) localization of the tumours strictly restricted to sympathetic structures with no localization in skin, liver, bone, or bone marrow which are the usual sites of metastases, (ii) metachronous development of the tumours and, when available, (iii) clear differences between the tumours' genomic profiles might help to specifically select the actual multifocal cases. The coexistence in the same patient of tumours with various histological types may also be taken into consideration.

Multiple malignancies also suggest a predisposition. Significantly high levels of $A L K$ expression in nervous tissues (retina, retinoblastomas, or medulloblastomas, for example Janoueix-Lerosey et $a l^{18}$ ) made this gene a good candidate particularly in patients with multiple nervous tumours. Furthermore, very recent data support the involvement of ALK point mutations in non-neuroblastic cancers. ${ }^{28}$ Conversely, no patient with various nervous tumours showed $A L K$ mutation and, reciprocally, none of our patients with germline mutation showed non-neuroblastic tumours. Hence, our results still suggest that the tumour spectrum of $A L K$ predisposing syndrome is tightly restricted to neuroblastic tumours.

Finally, the absence of $A L K$ mutations in three new pedigrees again emphasizes that other genes are most likely involved in the predisposition to neuroblastoma. Likewise, Mosse et al ${ }^{19}$ did not evidence any $A L K$ mutation in several kindred, strongly suggesting at least one other predisposing gene. Interestingly, the somatically acquired $A L K$ mutation in one familial case (Familial NB 2.2) indicates a probable synergistic effect between this potential unknown predisposing mutation and the secondary $A L K$ activation.

Another interesting conclusion of our work is the relatively low penetrance of $A L K$ mutations. Indeed, in $2 / 3$ apparently sporadic multifocal cases, the germline mutation is shared by three and five unaffected members of the family, respectively. A precise penetrance of $A L K$ mutation is hard to figure out, since many apparently unaffected carriers might in fact have had spontaneously regressive tumours, or asymptomatic stroma-rich tumours, as observed in two of the familial cases reported here (see Materials and Methods). Moreover, the penetrance is likely to be different from one specific mutation to another, although all occur within the kinase domain. Indeed, published data regarding affected and unaffected carriers suggest a $50 \%$ penetrance for $\mathrm{R} 1275 \mathrm{Q}$ and $\mathrm{R} 1192 \mathrm{P} A L K$ variants, the two main familial mutations. ${ }^{17,18}$ However, in our present series, the R1192P variant was associated with a much lower penetrance and an apparently sporadic presentation. Point mutations affecting the T1151 residue has also been reported in one sporadic case, ${ }^{22}$ and our present case again suggest a weak penetrance for this mutation. Modifier genes might also partly explain the differences within families or within individuals in a same family. They might also influence the severity of the tumour phenotype, from one single neuroblastoma to multiple diffuse tumours. Recently described polymorphisms associated with a higher risk of neuroblastoma, such as BARD1 variants or copy number variations at $1 \mathrm{q} 21$, might have such a role. Since the resolution of our array did not allow to analyse these loci, specific investigations with SNP arrays might further document this hypothesis. Finally, it is striking that, although constituting 35\% of somatic alterations, ${ }^{22}$ F1174 variants are again not observed in our germline analysis. This again illustrates that mutations that most strongly activate the tyrosine kinase domain might not be observed in patients without severe malformative phenotype. ${ }^{29}$

Altogether, our results show that germline $A L K$ mutations are rarely involved in sporadic neuroblastomas. Thus, systematic screening may not be proposed in non-familial cases. Nevertheless, among sporadic presentations, patients with multifocal tumours might be the best candidates for germline screening. Whenever a variant is observed in the tumour and then confirmed in the germline, germline screening, and radiological assessment (chest X-ray and ultrasonography) may 

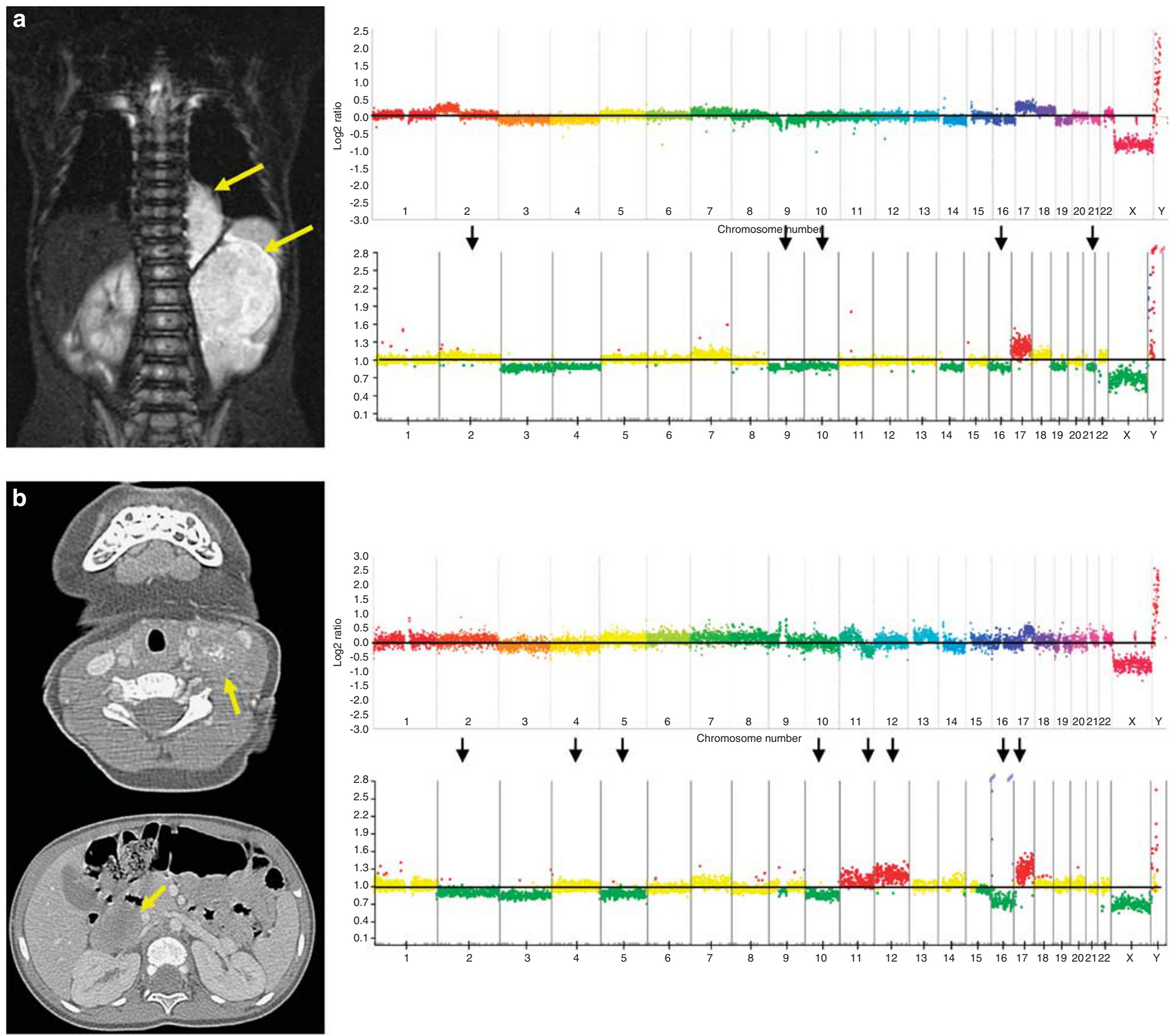

c
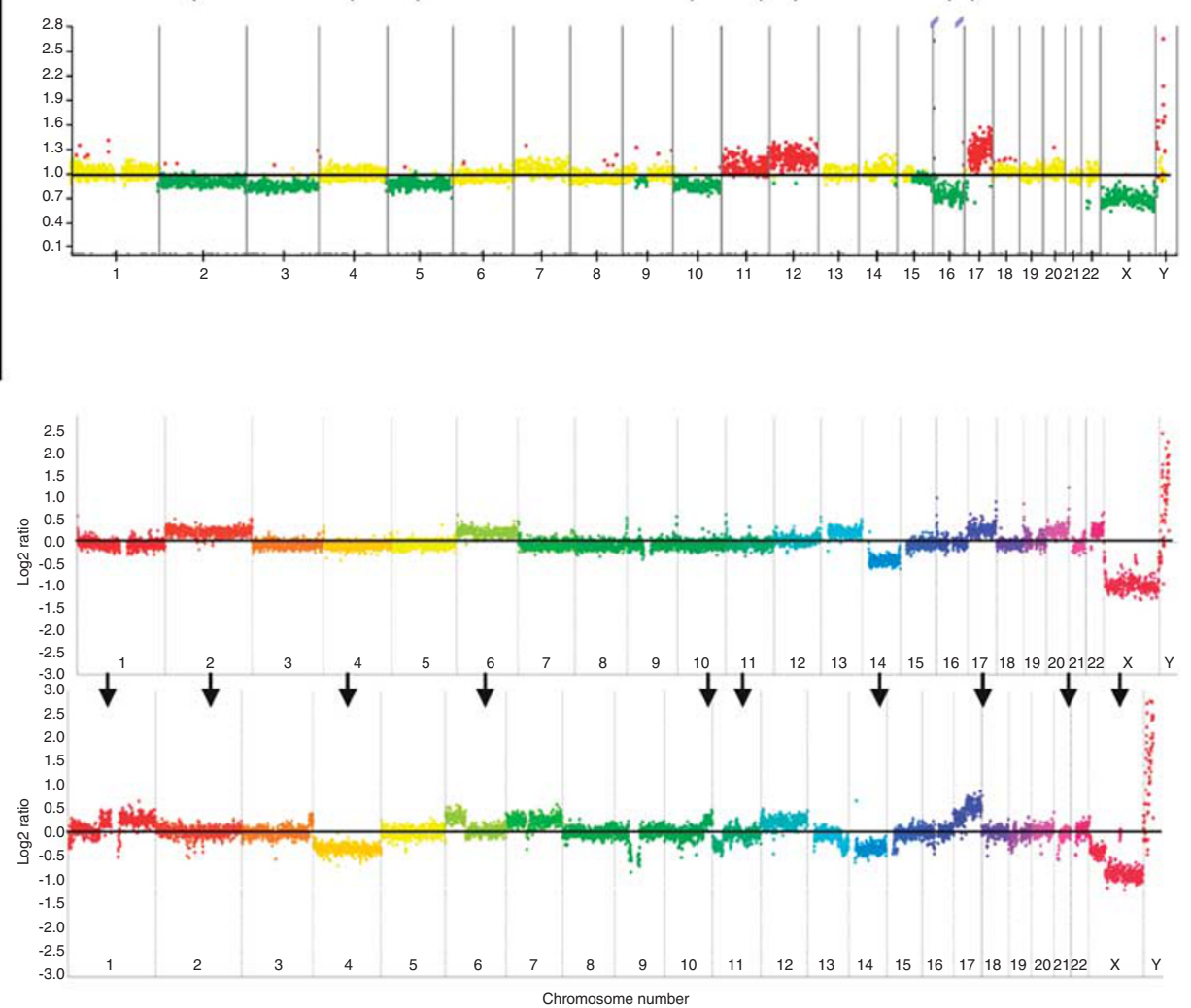

Figure 4 Multifocal neuroblastomas without neither ALK nor PHOX2B mutations. (a) Frontal view on whole body MRI (patient mNB11) showing a thoracic paravertebral tumour and a large adrenal gland tumour. The two patterns obtained by array CGH showed some common alterations and several differences (arrows). On BAC arrays, yellow indicates a normal copy number; red indicates a chromosomal gain, and green a chromosomal loss. (b) Cervical and abdominal tumours in patient mNB12; the two array CGH show clearly distinct genotypes (arrows). (c) Array CGH from the right (upper panel) and left (lower panel) adrenal neuroblastomas of the patient mNB3 showing an asynchronous bilateral tumour. 
be proposed to parents and siblings, given the low penetrance and frequent asymptomatic stroma-rich tumours. For asymptomatic carriers, we are currently proposing abdominal ultrasonography and urinary catecholamines dosage every 3 months, plus chest X-ray every 6 months up to 6 years of age. However, the presumable benefit of presymptomatic screening for young relatives of a mutation carrier still warrants further prospective survey.

\section{CONFLICT OF INTEREST}

The authors declare no conflict of interest.

\section{ACKNOWLEDGEMENTS}

This work was supported by grants from the Institut National du Cancer (INCa) and Direction de l'Hospitalisation et de l'Organisation des Soins (DHOS), the Ligue Nationale contre le Cancer (Equipe labellise'e and CIT project), the APAESIC (Association des Parents et des Amis des Enfants Soignés à l'Institut Curie), the Association Hubert Gouin, les amis de Claire, Les Bagouz à Manon, la Hulotte and Enfance et Sante. We thank all the clinicians and pathologists from the French Society of Cancer in children (SFCE) who refer their tumours and clinical data. We thank Dr Virginie Verkarre for the picture of the myenteric plexus hyperplasia.

$1 \mathrm{Li}$ FP, Fraumeni Jr JF: Rhabdomyosarcoma in children: epidemiologic study and identification of a familial cancer syndrome. J Natl Cancer Inst 1969; 43: 1365-1373.

2 Knudson Jr AG: Mutation and cancer: statistical study of retinoblastoma. Proc Natl Acad Sci USA 1971; 68: 820-823.

3 DeBaun MR, Niemitz EL, McNeil DE, Brandenburg SA, Lee MP, Feinberg AP. Epigenetic alterations of $\mathrm{H} 19$ and LIT1 distinguish patients with Beckwith-Wiedemann syndrome with cancer and birth defects. Am J Hum Genet 2002; 70: 604-611.

4 Scott RH, Douglas J, Baskcomb L et al: Constitutional $11 \mathrm{p} 15$ abnormalities, including heritable imprinting center mutations, cause nonsyndromic Wilms tumor. Nat Genet 2008; 40: 1329-1334.

5 Lim DH, Maher ER: Genomic imprinting syndromes and cancer. Adv Genet 2010; 70: $145-175$.

6 Brichard B, Heusterspreute M, De Potter $\mathrm{P}$ et al: Unilateral retinoblastoma, lack of familial history and older age does not exclude germline RB1 gene mutation. Eur J Cancer 2006; 42: 65-72.

7 Garre ML, Cama A, Bagnasco F et al: Medulloblastoma variants: age-dependent occurrence and relation to Gorlin syndrome-a new clinical perspective. Clin Cancer Res 2009; 15: 2463-2471.

8 Knudson Jr AG, Strong LC: Mutation and cancer: neuroblastoma and pheochromocytoma. Am J Hum Genet 1972; 24: 514-532.
9 Maris JM, Kyemba SM, Rebbeck TR et al: Molecular genetic analysis of familial neuroblastoma. Eur J Cancer 1997; 33: 1923-1928.

10 Maris JM, Kyemba SM, Rebbeck TR et al: Familial predisposition to neuroblastoma does not map to chromosome band 1p36. Cancer Res 1996; 56: 3421-3425.

11 Amiel J, Laudier B, Attie-Bitach T et al: Polyalanine expansion and frameshift mutations of the paired-like homeobox gene PHOX2B in congenital central hypoventilation syndrome. Nat Genet 2003; 33: 459-461.

12 Trochet $\mathrm{D}$, Bourdeaut $\mathrm{F}$, Janoueix-Lerosey I et al: Germline mutations of the paired-like homeobox 2B (PHOX2B) gene in neuroblastoma. Am J Hum Genet 2004; 74: 761-764.

13 Mosse YP, Laudenslager M, Khazi D et al: Germline PHOX2B mutation in hereditary neuroblastoma. Am J Hum Genet 2004; 75: 727-730.

14 Berry-Kravis EM, Zhou L, Rand CM, Weese-Mayer DE: Congenital central hypoventilation syndrome: PHOX2B mutations and phenotype. Am J Respir Crit Care Med 2006; 174: 1139-1144.

15 McConville C, Reid S, Baskcomb L, Douglas J, Rahman N: PHOX2B analysis in non-syndromic neuroblastoma cases shows novel mutations and genotype-phenotype associations. Am J Med Genet A 2006; 140: 1297-1301.

16 Raabe EH, Laudenslager M, Winter $\mathrm{C}$ et al: Prevalence and functional consequence of PHOX2B mutations in neuroblastoma. Oncogene 2008; 27: 469-476.

17 van Limpt V, Schramm A, van Lakeman A et al: The Phox2B homeobox gene is mutated in sporadic neuroblastomas. Oncogene 2004; 23: 9280-9288.

18 Janoueix-Lerosey I, Lequin D, Brugieres $\mathrm{L}$ et al: Somatic and germline activating mutations of the ALK kinase receptor in neuroblastoma. Nature 2008. 455. 967-970.

19 Mosse YP, Laudenslager M, Longo L et al: Identification of ALK as a major familial neuroblastoma predisposition gene. Nature 2008; 455: 930-935.

20 Caren $\mathrm{H}$, Abel F, Kogner $\mathrm{P}$, Martinsson T: High incidence of DNA mutations and gene amplifications of the ALK gene in advanced sporadic neuroblastoma tumours. Biochem J 2008; 416: 153-159.

21 Chen Y, Takita J, Choi YL et al: Oncogenic mutations of ALK kinase in neuroblastoma. Nature 2008; 455: 971-974.

22 De Brouwer S, De Preter K, Kumps C et al: Meta-analysis of neuroblastomas reveals a skewed ALK mutation spectrum in tumors with MYCN amplification. Clin Cancer Res 2010; 16: 4353-4362.

23 George RE, Sanda T, Hanna M et al: Activating mutations in ALK provide a therapeutic target in neuroblastoma. Nature 2008; 455: 975-978.

24 Roshkow JE, Haller JO, Berdon WE, Sane SM: Hirschsprung's disease, Ondine's curse, and neuroblastoma-manifestations of neurocristopathy. Pediatr Radiol 1988; 19: 45-49.

25 Kushner BH, Gilbert F, Helson L: Familial neuroblastoma. Case reports, literature review, and etiologic considerations. Cancer 1986; 57: 1887-1893.

26 Abbaszadeh F, Barker KT, McConville C, Scott RH, Rahman N: A new familial cancer syndrome including predisposition to Wilms tumor and neuroblastoma. Fam Cancer 2010; 9: 425-430.

27 Matera I, Bachetti T, Puppo F et al: PHOX2B mutations and polyalanine expansions correlate with the severity of the respiratory phenotype and associated symptoms in both congenital and late onset Central Hypoventilation syndrome. J Med Genet 2004; 41: 373-380.

28 Murugan AK, Xing M: Anaplastic thyroid cancers harbor novel oncogenic mutations of the ALK gene. Cancer Res 2011; 71: 4403-4411.

29 de Pontual L, Kettaneh D, Gordon C et al: Germline gain-of-function mutations of ALK disrupt central nervous system development. Hum Mutat 2011; 32: 272-276. 